\title{
Are minimum volume standards appropriate for lung and esophageal surgery?
}

\author{
Sebron Harrison, MD, ${ }^{\text {add }}$ Virginia Tangel, MA, ${ }^{\mathrm{c}}$ Xian Wu, MPH, ${ }^{\mathrm{b}}$ Paul Christos, DrPH, MS, \\ Licia Gaber-Baylis, BA, ${ }^{\mathrm{c}}$ Zachary Turnbull, MD ${ }^{\mathrm{c}}{ }^{\mathrm{Jeff}}$ Port, MD, ${ }^{\mathrm{a}}$ Nasser Altorki, MD, ${ }^{\mathrm{a}}$ and \\ Brendon Stiles, $\mathrm{MD}^{\mathrm{a}}$
}

\section{ABSTRACT}

Background: Several medical systems have adopted minimum volume standards for surgical procedures, including lung and esophageal resection. We sought to determine whether these proposed hospital cutoffs are associated with differences in outcomes.

Methods: Analyzing the State Inpatient Databases and Healthcare Cost and Utilization Project of the Agency for Healthcare Research and Quality, we evaluated all patients (aged $\geq 18$ years) who underwent lobectomy/pneumonectomy or esophagectomy for cancer in California, Florida, and New York (2009-2011). Hospitals were defined as low volume for each procedure per proposed minimum volume standards by year: $<40$ lung resections and $<20$ esophagectomies. We compared demographic data and determined the incidence of complications and mortality between patients operated on at low-versus high-volume hospitals. Propensity matching (of demographic characteristics, income, payer, and comorbidities) was performed to balance the cohorts for analysis.

Results: During the time period, 20,138 patients underwent lobectomy/ pneumonectomy of which 12,432 operations $(61.7 \%)$ were performed at low-volume hospitals $(n=456)$ and 7706 operations were performed at high-volume hospitals $(\mathrm{n}=48)$. Of 1324 patients undergoing esophagectomy, 1087 operations $(82.1 \%)$ were performed at low-volume hospitals $(\mathrm{n}=184)$, whereas only 237 operations were at high-volume hospitals $(n=6)$. After propensity matching (lung 1:1 and esophagus 2:1), no major differences were apparent for in-hospital mortality nor major complications for either lung or esophageal resection. Length of stay was longer in low-volume hospitals after lung resection (median 6 vs 5 days; $P<.001$ ), but not after esophageal resection.

Discussion: Although several groups have publicly called for minimum volume requirements for surgical procedures, the majority of patients undergo lung and esophageal resection at hospitals below the proposed cutoffs. The proposed standards for lung and esophageal resection are not associated with a difference in outcomes in this large administrative database. Efforts should be made to determine more meaningful minimum volume requirements and to determine whether such standards are appropriate. (J Thorac Cardiovasc Surg 2018;155:2683-94)

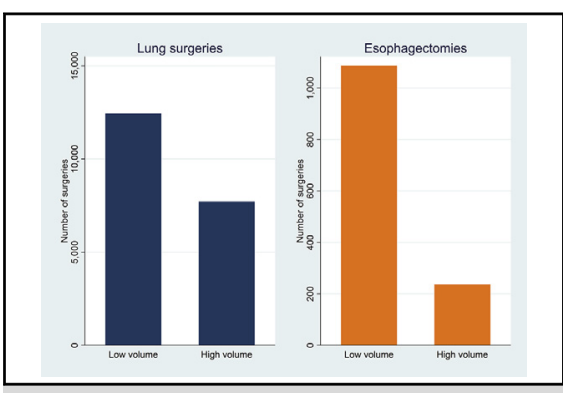

Propensity-matched outcomes for lung and esophageal surgery $(\%$ masked per Healthcare Cost and Utilization Project rules).

\section{Central Message}

Surgical volume alone does not account for differences in outcomes between high- and low-volume centers.

Perspective

Low-volume hospitals provide care to a substantial number of patients. The demographic characteristics of these patients are significantly different from those of patients who present to other centers. These differences likely factor into inferior outcomes at low-volume centers, and therefore volume alone should not be used to prevent centers from performing surgical procedures.

See Editorial Commentary page 2695

\footnotetext{
From the ${ }^{\mathrm{a}}$ Division of Thoracic Surgery, Department of Cardiothoracic Surgery, and ${ }^{b}$ Department of Healthcare Policy and Research, Weill Cornell Medicine, New York Presbyterian Hospital, New York, NY; ${ }^{\mathrm{c}}$ Department of Anesthesiology, Center for Perioperative Outcomes, Weill Cornell Medicine, New York, NY; and ${ }^{\mathrm{d}}$ New York-Presbyterian Brooklyn Methodist Hospital, New York, NY.

Dr Christos and Mr Wu were partially supported by grant No. UL1-TR000457-06 from the Clinical and Translational Science Center at Weill Cornell Medical College.
}

Read at the 97th Annual Meeting of The American Association for Thoracic Surgery, Boston, Massachusetts, April 29-May 3, 2017.

Received for publication July 18, 2017; revisions received Nov 10, 2017; accepted for publication Nov 16, 2017; available ahead of print Jan 19, 2018.

Address for reprints: Sebron Harrison, MD, Division of Thoracic Surgery, Department of Cardiothoracic Surgery, Weill Cornell Medicine, 525 E 68th St Ste M404, New York, NY 10065 (E-mail: swh9002@med.cornell.edu). $0022-5223 / \$ 36.00$

Copyright (c) 2017 by The American Association for Thoracic Surgery https://doi.org/10.1016/j.jtcvs.2017.11.073 


$\begin{array}{ll}\text { Abbreviations and Acronyms } \\ \text { HCUP }= & \text { Healthcare Cost and Utilization } \\ & \text { Project } \\ \text { ICD-9-CM = } & \text { International Classification of } \\ & \text { Diseases, Ninth Revision, Clinical } \\ & \text { Modification } \\ \text { LOS } \quad=\text { length of stay } \\ \text { POA }=\text { present on admission } \\ \text { SID }= & \text { state inpatient databases } \\ & \end{array}$

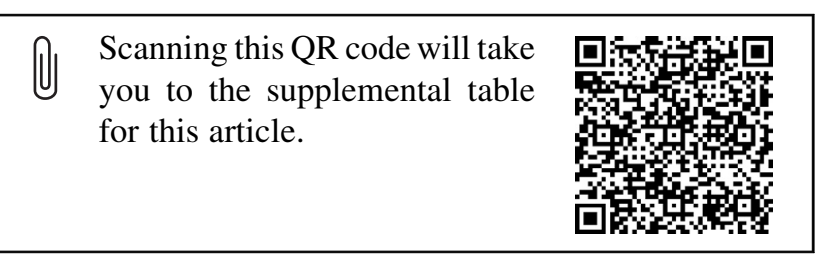

Outcomes following surgical procedures have become increasingly scrutinized. Debate exists regarding the influence surgical volume has on perioperative outcomes and on long-term survival. Seminal articles have described a relationship between hospitals performing higher numbers of complex thoracic surgical procedures, including lobectomy and esophagectomy, and improved short-term outcomes. ${ }^{1,2}$ However, subsequent work has questioned the methodologic rigor of these articles and has raised doubts as to whether surgical volume has an independent effect on outcomes. ${ }^{3-5}$ Nevertheless, hospital volume remains an appealing predictor of outcomes and has been used by high-profile groups, such as the Leapfrog Group and the Agency for Healthcare Research and Quality, as a surgical quality indicator. ${ }^{6-8}$ During 2015 , several hospital systems publicly announced a Take the Volume Pledge campaign in which they would restrict performance of 10 complex surgical procedures to hospitals who performed greater than a minimum threshold of cases. ${ }^{7}$ This initiative generated fierce debate related to both surgeon autonomy and to patient access to care. Apart from whether a true cause-and-effect relationship even exists between volume and outcomes, the methods used to derive cutpoints for minimum volumes for individual types of surgery were unclear. For example, minimum volumes proposed for esophageal cancer and lung cancer surgery were 20 and 40 cases, respectively. ${ }^{8}$ We sought to evaluate the effect of these volume cutpoints on perioperative outcomes following major lung resection and esophagectomy. To do so, we used data from large inpatient administrative databases. To account for potential selection bias in the relationship between surgical volume and outcomes, we applied a propensity score matching analysis to see whether volume effects on outcomes were independently present.

\section{METHODS \\ Study Database and Population}

We examined inpatient procedures and discharge data from adults (aged $\geq 18$ years) using 2009 to 2011 data from California, Florida, and New York from state inpatient databases (SIDs), and the Healthcare Cost and Utilization Project (HCUP) of the Agency for Healthcare Research and Quality. ${ }^{9}$ SIDs contain administrative inpatient data from nonfederal, nonpsychiatric hospitals. Each inpatient hospital admission corresponds to 1 individual record. Variables available for each admission include demographic information; International Classification of Diseases, Ninth Revision, Clinical Modification (ICD-9-CM) codes for diagnoses and procedures; admission and discharge dates; and discharge disposition. Measures of procedure volume by hospital and hospital length of stay (LOS) can be calculated with available data. Data on individual surgeons was not available for California. SIDs contain indicators for each diagnosis, allowing for differentiation between present-on-admission (POA) status and not POA for each diagnosis. This provides the ability to distinguish preexisting medical comorbidities from intraoperative and procedural complications. Data on hospital charges were adjusted to 2016 US dollars.

The Weill Cornell Medicine Institutional Review Board approved all study activities. Using ICD-9-CM procedure codes, we retrospectively identified records from January 2009 through December 2011 of patients undergoing lobectomy, pneumonectomy, or esophagectomy surgeries (ICD-9-CM procedure codes 324 for lobectomy, 325 for pneumonectomy, and 424 for esophagectomy). Only records with identified lung or esophageal cancer were included (ICD-9-CM diagnosis codes 162 and 150 , respectively). Lobectomy and pneumonectomy surgeries were grouped together to form a lung resection procedure category. Hospital volume for lung and esophageal surgeries were calculated separately, grouping hospitals by the total number of surgeries performed by year during the study period. Categorizations of hospital volume into high and low categories were based on criteria established by the Leapfrog Group. ${ }^{9}$ Two separate analyses were conducted: 1 comparing outcomes in hospitals performing $<40$ lung surgeries (low volume) each year versus 40 or more lung surgeries (high volume) each year during the 3 -year period. The other analyses compared outcomes in hospitals performing fewer than 20 esophagectomies (low volume) each year versus 20 or more esophagectomies (high volume) each year during the 3-year period. Because the study period occurred over 3 years, a hospital could appear in both low and high volume categories, if it performed fewer than 40 lung resections in 2009 and 40 or more in 2010 and 2011, for example.

\section{Primary Outcomes}

The primary outcomes of our study were the rates of in-hospital mortality, hospital LOS, and the presence of postoperative complications. In-hospital mortality was defined by an existing HCUP variable. LOS represented the number of days between visit admission and visit discharge. Intraoperative and postoperative complications of interest included pulmonary, wound, infectious, urinary, gastrointestinal, cardiovascular, systemic, and intraoperative/procedural complications (a full list of complications is listed in Table E1). Although ICD-9-CM codes have been shown to be an imprecise way to measure complications, ${ }^{10,11}$ they are the only way one can ascertain such outcomes in the SID.

\section{Statistical Analysis}

Demographic characteristics and POA comorbidities were summarized by high- and low- volume hospitals for lung and esophageal surgeries, respectively. We calculated descriptive statistics using frequency and proportions for categorical variables and medians and interquartile ranges for continuous variables.

To account for potential selection bias in the relationship between surgical volume and outcomes, we applied a propensity score matching analysis. The propensity score is the probability of a patient treated in a 
TABLE 1. Unadjusted demographic and present-on-admission diagnoses in patients undergoing lung resections

\begin{tabular}{|c|c|c|c|c|}
\hline & \multicolumn{4}{|c|}{ Unadjusted } \\
\hline & $\frac{\text { Low volume: } 1-39 \text { surgeries } / \mathbf{y}}{n(\%)^{*}}$ & $\frac{\text { High volume: } 40+\text { surgeries } / \mathbf{y}}{\mathrm{n}(\%)^{*}}$ & $P$ value & $\frac{\text { Standardized difference }}{\%}$ \\
\hline Total & $12,432(61.7)$ & $7706(38.3)$ & & \\
\hline Year & & & .1464 & \\
\hline 2009 & $4283(34.5)$ & $2566(33.3)$ & & 2.4 \\
\hline 2010 & 4055 (32.6) & $2604(33.8)$ & & -2.5 \\
\hline 2011 & $4094(32.9)$ & $2536(32.9)$ & & 0 \\
\hline State & & & $<.01$ & \\
\hline California & 5495 (44.2) & $1352(17.5)$ & & 60.3 \\
\hline Florida & $4356(35)$ & $2681(34.8)$ & & 0.5 \\
\hline New York & $2581(20.8)$ & $3673(47.7)$ & & -59.1 \\
\hline Age category (y) & & & .0213 & \\
\hline $18-54$ & $1285(10.3)$ & $838(10.9)$ & & -1.7 \\
\hline $55-64$ & $2767(22.3)$ & $1813(23.5)$ & & -3.0 \\
\hline $65-74$ & 4839 (38.9) & $2992(38.8)$ & & 0.2 \\
\hline$\geq 75$ & $3541(28.5)$ & $2063(26.8)$ & & 3.8 \\
\hline Gender & & & .3038 & \\
\hline Male & $5860(47.1)$ & $3575(46.4)$ & & 1.5 \\
\hline Female & $6572(52.9)$ & $4131(53.6)$ & & -1.5 \\
\hline $\begin{array}{l}\text { Median household income state } \\
\text { quartile for patient ZIP code }\end{array}$ & & & $<.01$ & \\
\hline First & $2744(22.1)$ & $1248(16.2)$ & & 15.0 \\
\hline Second & $3158(25.4)$ & $1676(21.7)$ & & 8.6 \\
\hline Third & $3171(25.5)$ & $2117(27.5)$ & & -4.5 \\
\hline Fourth & $3070(24.7)$ & $2495(32.4)$ & & -17.1 \\
\hline Missing & $289(2.3)$ & $170(2.2)$ & & 0.8 \\
\hline Race & & & $<.01$ & \\
\hline Nonwhite & $2803(22.5)$ & $1157(15.0)$ & & 19.4 \\
\hline White & $9455(76.1)$ & $6496(84.3)$ & & -20.8 \\
\hline Missing & $174(1.4)$ & $53(0.7)$ & & 7.0 \\
\hline Procedure type & & & .0229 & \\
\hline Lobectomy & $11,703(94.1)$ & $7193(93.3)$ & & 3.3 \\
\hline Pneumonectomy & $729(5.9)$ & $513(6.7)$ & & -3.3 \\
\hline Payer (revised) & & & $<.01$ & \\
\hline Medicare/Medicaid & 8875 (71.4) & $5060(65.7)$ & & 12.4 \\
\hline Private insurance & $3161(25.4)$ & $2498(32.4)$ & & -15.5 \\
\hline Other, including missing & $396(3.2)$ & $148(1.9)$ & & 8.0 \\
\hline Modified Charlson index, 3 categories & & & $<.01$ & \\
\hline 2 & $4748(38.2)$ & $3182(41.3)$ & & -6.3 \\
\hline 3 & $4536(36.5)$ & $2404(31.2)$ & & 11.2 \\
\hline $4+$ & $3148(25.3)$ & $2120(27.5)$ & & -5.0 \\
\hline $\begin{array}{l}\text { Chronic obstructive pulmonary disease } \\
\text { present on admission }\end{array}$ & $5528(44.5)$ & $2917(37.9)$ & $<.01$ & 13.5 \\
\hline Peripheral vascular disorders & $1029(8.3)$ & $528(6.9)$ & .0002 & 5.4 \\
\hline Hypertension, uncomplicated & $6703(53.9)$ & 4049 (52.5) & .0575 & 2.8 \\
\hline Hypertension, complicated & $873(7)$ & $312(4)$ & $<.01$ & 13.0 \\
\hline Chronic pulmonary disease & $5957(47.9)$ & $3238(42)$ & $<.01$ & 11.9 \\
\hline Diabetes, uncomplicated & $2148(17.3)$ & $1108(14.4)$ & $<.01$ & 8.0 \\
\hline Renal failure & $768(6.2)$ & $335(4.3)$ & $<.01$ & 8.2 \\
\hline Metastatic cancer & $2150(17.3)$ & 1669 (21.7) & $<.01$ & -11.0 \\
\hline
\end{tabular}


TABLE 1. Continued

\begin{tabular}{|c|c|c|c|c|}
\hline & \multicolumn{4}{|c|}{ Unadjusted } \\
\hline & Low volume: $1-39$ surgeries/y & High volume: $40+$ surgeries/y & & $\underline{\text { Standardized difference }}$ \\
\hline & $\begin{array}{c}\mathbf{n}(\%)^{*} \\
\end{array}$ & 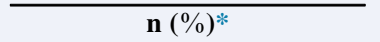 & $P$ value & $\%$ \\
\hline Obesity & $970(7.8)$ & $453(5.9)$ & $<.01$ & 7.6 \\
\hline Deficiency anemia & $1283(10.3)$ & $455(5.9)$ & $<.01$ & 16.2 \\
\hline Depression & $948(7.6)$ & $523(6.8)$ & .0262 & 3.2 \\
\hline
\end{tabular}

*Percentages may not equal 100 due to rounding.

high-volume hospital given the baseline patient demographic and clinical characteristics. These demographic and clinical characteristics included year, state, age (categorical), gender, median household income in the patient's ZIP code (in quartiles) by state, race, payer, and POA comorbidities (as measured in a collapsed version of a modified Charlson index, ${ }^{12}$ including congestive heart failure, dementia, chronic pulmonary disease, rheumatologic disease, mild liver disease, diabetes with chronic complications, hemiplegia/paraplegia, renal disease, malignancy, moderate or severe liver disease, a metastatic solid tumor, or AIDS/ HIV). The propensity scores were calculated using logistic regression models for lung and esophageal surgeries, respectively. In a propensity score-matched analysis, patients were matched 1 to 1 for lung surgery and 1 to 2 for esophageal surgery. A greedy matching algorithm without replacement and with a caliper distance of 0.2 of the standard deviation of the logistic propensity score was used. The standardized mean difference assessed the balance of covariates between high-volume and low-volume groups before and after propensity score matching. To assess the association between surgical volume and outcomes in propensity-matched cohorts, conditional logistic regressions and the Wilcoxon signed rank-sum test were used. In addition, we constructed multilevel mixed-effects regression models, modeling the individual hospitals as a random effect by using a unique hospital identifier to account for clustering of patients within hospitals, and to account for the potential of individual hospitals appearing in both low- and high-volume categories over the 3-year period. Results from the regression models were reported as odds ratios (ORs) with $95 \%$ confidence intervals (CIs). All results reported are from conditional logistic regression models, unless otherwise noted.

All $P$ values are 2 -sided with statistical significance evaluated at an alpha level of 0.05. All statistical analyses were performed using SAS version 9.3 (SAS Institute, Cary, NC).

\section{RESULTS}

\section{Lung Resection (Lobectomy and Pneumonectomy)}

There were a total of 20,138 lung resections between 2009 and 2011 in California, Florida, and New York. Among these, $12,432(61.7 \%)$ were performed at low-volume hospitals and the remaining 7706 (38.3\%) were performed at high-volume hospitals (Table 1). The number of lung resections significantly differed by age, with low-volume hospitals having a slightly higher proportion of patients older than age 65 years $(67.4 \%$ vs $65.6 \% ; P=.02$ ). High-volume hospitals had a greater proportion of privately insured $(32.4 \%$ vs $25.4 \% ; P<.01)$ and white patients $(84.3 \%$ vs $76.1 \%$; $P<.01)$. In addition, a greater proportion of patients undergoing lung resections at high-volume hospitals lived in ZIP codes with median household incomes in the top quartile of their respective state $(32.4 \%$ vs $24.7 \%$; $P<.01)$.
The proportion of patients with more comorbidities (scores of $4+$ on the modified Charlson index vs separate categories of 2 and 3) was slightly higher in the highvolume hospital category $(27.5 \%$ vs $25.3 \% ; P<.01)$. However, patients with individual comorbidities (eg, chronic obstructive pulmonary disease, peripheral vascular disorders, complicated hypertension, uncomplicated diabetes, renal failure, obesity, deficiency anemia, and depression) were all significantly more likely to undergo a lung resection at a low-volume hospital $(P<.01$ for all except depression, which was $P<.03$ ).

In-hospital mortality for lung resections in the unadjusted data was significantly higher at low-volume hospitals $(2.2 \%$ vs $1.7 \% ; P=.01)$, as was the presence of any postoperative complication $(45.5 \%$ vs $42.7 \% ; P<.01)$. The median hospital LOS was also longer in patients undergoing lung resections at low-volume hospitals (6 days [range, 4-9 days] vs 5 days [range, 4-7 days]; $P<.01)$. Finally, median total charges incurred during the hospital stay were markedly higher for patients undergoing surgery in low-volume hospitals $(\$ 99,097$ [range, \$64,846-\$150,996] vs \$68,164 [range, \$50,307$\$ 99,729$ ]; $P<.01)$.

\section{Esophagectomy}

There were a total of 1324 esophagectomies performed between 2009 and 2011 in California, Florida, and New York, the majority of which $(\mathrm{n}=1087 ; 82.1 \%)$ were performed at low-volume hospitals (Table 2). The remaining $237(17.9 \%)$ were performed at high-volume hospitals. There was a significantly higher proportion of white patients undergoing esophagectomy at high-volume hospitals $(89.9 \%$ vs $79.3 \% ; P<.01)$, as well as a higher proportion of privately insured patients at high-volume hospitals $(52.7 \%$ vs $40.8 \% ; P<.01)$. The proportion of patients with 4 or more comorbidities on the modified Charlson index was higher in low-volume hospitals $(27 \%$ vs $16.9 \% ; P<.01)$, as were POA diagnoses of weight loss $(10.1 \%$ vs $4.6 \% ; P<.01)$ and deficiency anemia $(12.9 \%$ vs $4.6 \% ; P<.01)$.

In the unadjusted data, there were no significant differences in in-hospital mortality following esophagectomy between high- and low-volume hospitals $(P=.48$; not shown). However, patients treated at low-volume 
TABLE 2. Unadjusted demographic and present-on-admission diagnoses in patients undergoing esophagectomy

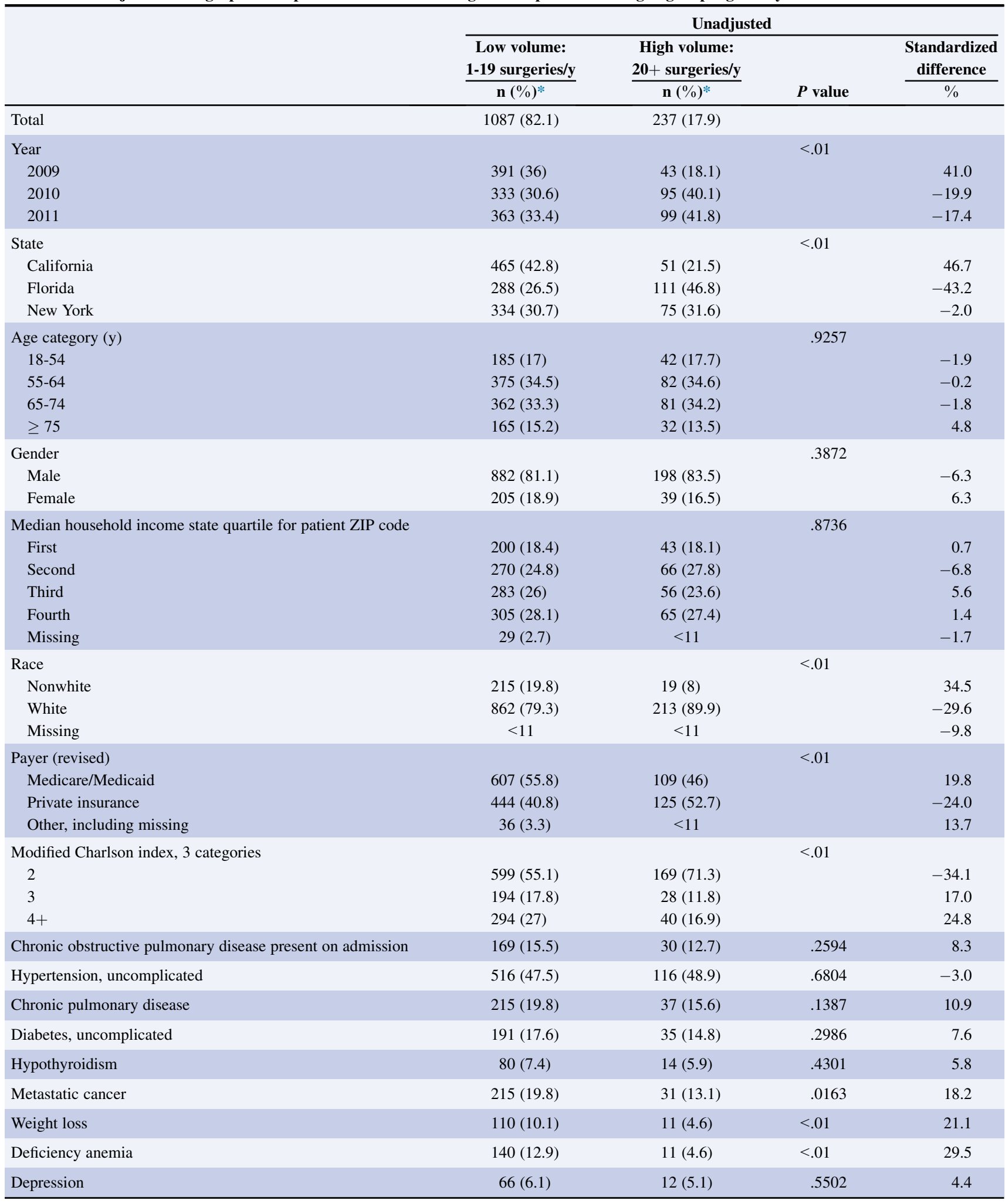

*Percentages may not equal 100 due to rounding. 
TABLE 3. Adjusted demographic and present-on-admission diagnoses (lung resections)

\begin{tabular}{|c|c|c|c|c|}
\hline & \multicolumn{4}{|c|}{ Lung surgeries: Propensity score matched } \\
\hline & $\begin{array}{c}\begin{array}{c}\text { Low volume: } \\
\text { 1-39 surgeries/y }\end{array} \\
\mathbf{n}(\%)^{*}\end{array}$ & $\begin{array}{c}\text { High volume: } \\
\frac{40+\text { surgeries/y }}{\mathbf{n}(\%)^{*}}\end{array}$ & $P$ value & $\begin{array}{c}\begin{array}{c}\text { Standardized } \\
\text { difference }\end{array} \\
\%\end{array}$ \\
\hline Total & $6349(50.0)$ & $6349(50.0)$ & & \\
\hline Year & & & .1569 & \\
\hline 2009 & $2196(34.6)$ & $2133(33.6)$ & & 2.1 \\
\hline 2010 & $2037(32.1)$ & $2138(33.7)$ & & -3.4 \\
\hline 2011 & $2116(33.3)$ & $2078(32.7)$ & & 1.3 \\
\hline State & & & .1222 & \\
\hline California & $1436(22.6)$ & $1352(21.3)$ & & 3.2 \\
\hline Florida & $2566(40.4)$ & 2659 (41.9) & & -3.0 \\
\hline New York & $2347(37.0)$ & $2338(36.8)$ & & 0.3 \\
\hline Age category $(y)$ & & & .9342 & \\
\hline $18-54$ & $680(10.7)$ & $671(10.6)$ & & 0.5 \\
\hline $55-64$ & $1432(22.6)$ & $1409(22.2)$ & & 0.9 \\
\hline $65-74$ & $2462(38.8)$ & $2492(39.3)$ & & -1.0 \\
\hline$\geq 75$ & $1775(28.0)$ & $1777(28.0)$ & & -0.1 \\
\hline Gender & & & .6828 & \\
\hline Male & $3017(47.5)$ & $3040(47.9)$ & & -0.7 \\
\hline Female & $3332(52.5)$ & $3309(52.1)$ & & 0.7 \\
\hline Median household income state quartile for patient ZIP code & & & .4562 & \\
\hline First & $1139(17.9)$ & $1168(18.4)$ & & -1.2 \\
\hline Second & $1518(23.9)$ & $1535(24.2)$ & & -0.6 \\
\hline Third & $1714(27.0)$ & $1763(27.8)$ & & -1.7 \\
\hline Fourth & $1820(28.7)$ & $1726(27.2)$ & & 3.3 \\
\hline Missing & $158(2.5)$ & $157(2.5)$ & & 0.1 \\
\hline Race & & & .6506 & \\
\hline Nonwhite & $1072(16.9)$ & $1104(17.4)$ & & -1.3 \\
\hline White & $5231(82.4)$ & $5194(81.8)$ & & 1.5 \\
\hline Missing & $46(0.7)$ & $51(0.8)$ & & -0.9 \\
\hline Procedure type & & & .9133 & \\
\hline Lobectomy & $5942(93.6)$ & 5945 (93.6) & & -0.2 \\
\hline Pneumonectomy & $407(6.4)$ & $404(6.4)$ & & 0.2 \\
\hline Payer (revised) & & & .397 & \\
\hline Medicare/Medicaid & $4383(69.0)$ & $4446(70.0)$ & & -2.2 \\
\hline Private insurance & $1809(28.5)$ & $1761(27.7)$ & & 1.7 \\
\hline Other, including missing & $157(2.5)$ & $142(2.2)$ & & 1.6 \\
\hline Modified Charlson index, 3 categories & & & .5191 & \\
\hline 2 & $2560(40.3)$ & $2578(40.6)$ & & -0.6 \\
\hline 3 & $2136(33.6)$ & $2173(34.2)$ & & -1.2 \\
\hline $4+$ & $1653(26.0)$ & $1598(25.2)$ & & 2.0 \\
\hline Chronic obstructive pulmonary disease present on admission & $2616(41.2)$ & $2629(41.4)$ & .8148 & -0.4 \\
\hline Peripheral vascular disorders & $468(7.4)$ & $471(7.4)$ & .9190 & -0.2 \\
\hline Hypertension, uncomplicated & $3409(53.7)$ & $3464(54.6)$ & .3273 & -1.7 \\
\hline Hypertension, complicated & $320(5.0)$ & $292(4.6)$ & .2460 & 2.1 \\
\hline Chronic pulmonary disease & $2840(44.7)$ & $2876(45.3)$ & .5208 & -1.1 \\
\hline Diabetes, uncomplicated & $968(15.3)$ & $1007(15.9)$ & .3396 & -1.7 \\
\hline Hypothyroidism & $702(11.1)$ & $710(11.2)$ & .8213 & -0.4 \\
\hline Renal failure & $313(4.9)$ & $300(4.7)$ & .5904 & 1.0 \\
\hline
\end{tabular}




\begin{tabular}{|c|c|c|c|c|}
\hline & \multicolumn{4}{|c|}{ Lung surgeries: Propensity score matched } \\
\hline & $\begin{array}{c}\begin{array}{c}\text { Low volume: } \\
\text { 1-39 surgeries/y }\end{array} \\
\mathbf{n}(\%)^{*}\end{array}$ & $\begin{array}{c}\text { High volume: } \\
\frac{40+\text { surgeries/y }}{n(\%)}\end{array}$ & $P$ value & $\begin{array}{c}\begin{array}{c}\text { Standardized } \\
\text { difference }\end{array} \\
\%\end{array}$ \\
\hline Metastatic cancer & $1234(19.4)$ & $1178(18.6)$ & .2052 & 2.2 \\
\hline Obesity & $417(6.6)$ & $399(6.3)$ & .5148 & 1.2 \\
\hline Deficiency anemia & $463(7.3)$ & $436(6.9)$ & .3502 & 1.7 \\
\hline Depression & $450(7.1)$ & $458(7.2)$ & .7829 & -0.5 \\
\hline Weight loss & & & & \\
\hline
\end{tabular}

*Percentages may not equal 100 due to rounding.

hospitals were significantly more likely to have a complication than those treated at high-volume centers $(67.8 \%$ vs $60.3 \% ; P=.03)$.

Median total charges were significantly higher at lowvolume hospitals $(\$ 178,950$ [range, $\$ 103,634-\$ 336,483$ ] vs $\$ 100,364$ [range, $\$ 75,299-\$ 293,327$ ]; $P<.01$ ).

\section{Propensity Score Matching}

Patients were propensity matched according to multiple characteristics to generate balanced groups of comparison in which to compare volume effects upon outcome (Tables 3 and 4). In the propensity-matched analysis for lung resection, there was no volume effect on in-hospital mortality when comparing low- and high-volume hospitals (OR, 1.19; 95\% CI, 0.93-1.54; $P=.17$ ) (Table 5). There was also no significant difference in postoperative complications (OR, 1.02; 95\% CI, 0.95-1.09; $P=.54$ ) (Table 5). However, differences between total hospital LOS remained significant, with low-volume hospitals having a longer median LOS than high-volume hospitals (6 days [interquartile range, 4-9 days] vs 5 days [range, 4-8 days]; $P<.01$ ). Lower-volume hospitals also had significantly higher charges than high-volume hospitals (\$82,529 [range, $\$ 54,953-\$ 126,991]$ vs $\$ 70,997$ [range, \$51,254$\$ 104,855] ; P<.01)$. However, when accounting for clustering at the hospital level, there was no longer a significant difference in charges between groups ( $P=.13$; not shown).

We also compared patients undergoing esophagectomy in a propensity-matched data set. In- hospital mortality was not significantly different $(P>.05)$ between patients undergoing esophagectomy at low- $(3.7 \%)$ and highvolume hospitals (percentage masked due to HCUP rules for reporting on $\mathrm{n}<11$ ). There was no significant difference in the presence of any complication between high- and lowvolume hospitals $(P=.27)$, or of rates of cardiovascular, pulmonary, infectious, or intraoperative complications or of anastomotic leaks (Table 6).

Differences in median LOS were also not different between low- and high-volume hospitals (median for low-volume hospitals: 11.5 days [range, 8-18 days] vs high-volume hospitals: 2 days [range, 9-19 days];
$P=.13)$, although the total hospital charges incurred were significantly higher for lower-volume hospitals than high-volume hospitals ( $\$ 146,849$ [range, 99,732-283,377] vs $\$ 125,882$ [range, $\$ 77,270-\$ 336,580$ ]). There was no difference in total hospital charges between volume groups when accounting for clustering within hospital $(P=.31$; not shown).

\section{DISCUSSION}

Much has been written to support the hypothesis that surgical volume affects perioperative outcomes, including thoracic surgical procedures. ${ }^{1-8,13-19}$ In 2001, Bach and colleagues $^{1}$ published the results of a large study of the Surveillance, Epidemiology, and End Result database that focused on surgery for lung cancer. Their results suggested not only that patients undergoing lung surgery at very-lowvolume hospitals did worse in the immediate postoperative period with higher rates of serious complications, but also that overall cancer-related survival was worse. In 2002, Birkmeyer and colleagues ${ }^{2}$ published their landmark study from an administrative database of Medicare patients undergoing 14 complex surgical procedures, including major lung resection and esophagectomy. That study concluded that overall, a patient's odds of survival were better in higher-volume centers. More recently, Finks and colleagues ${ }^{16}$ used Medicare Provider Analysis and Review files in an effort to further investigate volume effects on lung resection and esophagectomy, among other complex surgical procedures. The researchers ${ }^{17}$ found volumeattributable improvements in operative mortality in lung resections and esophagectomies.

These and similar studies have led health care providers and policy makers to advocate for regionalization or centralization of care in high-volume centers in an effort to improve mortality outcomes. ${ }^{18,19}$ However, whether regionalization based on surgical volume improves outcomes remains controversial. For example, a recent study reporting mortality outcomes in Canada following regionalization of thoracic surgery did not show improvement in 30-day mortality following esophagectomy or lobectomy. ${ }^{19}$ 
TABLE 4. Adjusted demographic and present-on-admission diagnoses (esophagectomies)

\begin{tabular}{|c|c|c|c|c|}
\hline & \multicolumn{4}{|c|}{ Esophagectomy: Propensity score matched } \\
\hline & $\begin{array}{c}\text { Low volume: } \\
\frac{1-19 \text { surgeries } / \mathbf{y}}{\mathbf{n}(\%)^{*}}\end{array}$ & $\begin{array}{c}\text { High volume: } \\
\frac{20+\text { surgeries } / \mathbf{y}}{\text { n }(\%)^{*}}\end{array}$ & $P$ value & $\begin{array}{c}\begin{array}{c}\text { Standardized } \\
\text { difference }\end{array} \\
\%\end{array}$ \\
\hline Total & $376(66.7)$ & $188(33.3)$ & & \\
\hline $\begin{array}{l}\text { Year } \\
2009 \\
2010 \\
2011\end{array}$ & $\begin{array}{r}74(19.7) \\
144(38.3) \\
158(42.0)\end{array}$ & $\begin{array}{l}42(22.3) \\
76(40.4) \\
70(37.2)\end{array}$ & .5254 & $\begin{array}{r}-6.5 \\
-4.4 \\
9.8\end{array}$ \\
\hline $\begin{array}{l}\text { State } \\
\text { California } \\
\text { Florida } \\
\text { New York }\end{array}$ & $\begin{array}{l}115(30.6) \\
130(34.6) \\
131(34.8)\end{array}$ & $\begin{array}{l}51(27.1) \\
77(41.0) \\
60(31.9)\end{array}$ & .3300 & $\begin{array}{r}7.6 \\
-13.2 \\
6.2\end{array}$ \\
\hline $\begin{array}{l}\text { Age category (y) } \\
18-54 \\
55-64 \\
65-74 \\
\geq 75\end{array}$ & $\begin{array}{r}64(17.0) \\
132(35.1) \\
120(31.9) \\
60(16.0)\end{array}$ & $\begin{array}{l}31(16.5) \\
72(38.3) \\
56(29.8) \\
29(15.4)\end{array}$ & .9014 & $\begin{array}{r}1.4 \\
-6.6 \\
4.6 \\
1.5\end{array}$ \\
\hline $\begin{array}{l}\text { Gender } \\
\text { Male } \\
\text { Female }\end{array}$ & $\begin{array}{r}306(81.4) \\
70(18.6)\end{array}$ & $\begin{array}{r}160(85.1) \\
28(14.9)\end{array}$ & .2713 & $\begin{array}{r}-10 \\
10\end{array}$ \\
\hline $\begin{array}{l}\text { Median household income state quartile for patient ZIP code } \\
\text { First } \\
\text { Second } \\
\text { Third } \\
\text { Fourth } \\
\text { Missing }\end{array}$ & $\begin{array}{r}55(14.6) \\
105(27.9) \\
89(23.7) \\
117(31.1) \\
10(2.7)\end{array}$ & $\begin{array}{c}38(20.2) \\
45(23.9) \\
48(25.5) \\
53(28.2) \\
4(2.1)\end{array}$ & .434 & $\begin{array}{r}14.8 \\
9.1 \\
-4.3 \\
6.4 \\
3.5\end{array}$ \\
\hline $\begin{array}{l}\text { Race } \\
\text { Nonwhite } \\
\text { White } \\
\text { Missing }\end{array}$ & $\begin{aligned} 38 & (10.1) \\
336 & (89.4) \\
2 & (0.5)\end{aligned}$ & $\begin{aligned} 19 & (10.1) \\
166 & (88.3) \\
3 & (1.6)\end{aligned}$ & .4458 & $\begin{array}{r}0 \\
3.4 \\
-10.4\end{array}$ \\
\hline $\begin{array}{l}\text { Procedure type } \\
\text { Lobectomy } \\
\text { Pneumonectomy }\end{array}$ & & & & \\
\hline $\begin{array}{l}\text { Payer (revised) } \\
\text { Medicare/Medicaid } \\
\text { Private insurance } \\
\text { Other, including missing }\end{array}$ & $\begin{array}{c}186(49.5) \\
185(49.2) \\
5(1.3)\end{array}$ & $\begin{array}{c}92(48.9) \\
93(49.5) \\
3(1.6)\end{array}$ & .9649 & $\begin{array}{r}1.1 \\
-0.5 \\
-2.2\end{array}$ \\
\hline $\begin{array}{l}\text { Modified Charlson index, } 3 \text { categories } \\
2 \\
3 \\
4+\end{array}$ & $\begin{array}{r}253(67.3) \\
49(13.0) \\
74(19.7)\end{array}$ & $\begin{array}{r}123(65.4) \\
28(14.9) \\
37(19.7)\end{array}$ & .8256 & $\begin{array}{r}3.9 \\
-5.4 \\
0\end{array}$ \\
\hline Chronic obstructive pulmonary disease present on admission & $46(12.2)$ & $27(14.4)$ & .478 & -6.3 \\
\hline \multicolumn{5}{|l|}{ Peripheral vascular disorders } \\
\hline Hypertension, uncomplicated & $185(49.2)$ & $89(47.3)$ & .6767 & 3.7 \\
\hline \multicolumn{5}{|l|}{ Hypertension, complicated } \\
\hline Chronic pulmonary disease & $59(15.7)$ & $34(18.1)$ & .4702 & -6.4 \\
\hline Diabetes, uncomplicated & $53(14.1)$ & $28(14.9)$ & .799 & -2.3 \\
\hline Hypothyroidism & $25(6.7)$ & $13(6.9)$ & .9055 & -1.1 \\
\hline Renal failure & & & & \\
\hline
\end{tabular}




\begin{tabular}{|c|c|c|c|c|}
\hline & \multicolumn{4}{|c|}{ Esophagectomy: Propensity score matched } \\
\hline & $\begin{array}{c}\begin{array}{c}\text { Low volume: } \\
\text { 1-19 surgeries/y }\end{array} \\
\text { n }(\%)^{*}\end{array}$ & $\begin{array}{c}\text { High volume: } \\
\frac{20+\text { surgeries/y }}{\text { n }(\%)^{*}}\end{array}$ & $P$ value & $\begin{array}{c}\begin{array}{c}\text { Standardized } \\
\text { difference }\end{array} \\
\%\end{array}$ \\
\hline Metastatic cancer & $62(16.5)$ & $29(15.4)$ & .7461 & 2.9 \\
\hline \multicolumn{5}{|l|}{ Obesity } \\
\hline Deficiency anemia & $18(4.8)$ & $11(5.9)$ & .5897 & -4.7 \\
\hline Depression & $20(5.3)$ & $11(5.9)$ & .7939 & -2.3 \\
\hline Weight loss & $17(4.5)$ & $10(5.3)$ & .6757 & -3.7 \\
\hline
\end{tabular}

*Percentages may not equal 100 due to rounding.

Despite the intuitive appeal of using surgical volume as a predictor of surgical outcomes and as a quality indicator, the methodologic rigor of many volume-outcome studies has been questioned. ${ }^{3,4}$ Several concerns have been raised about these studies, including the tendency to place procedure volumes into arbitrarily defined categories, the overreliance on administrative datasets for most studies, and the failure to account for the effect of other factors associated with surgical volume on outcome. Some have therefore suggested that volume effects upon surgical outcomes are simply associative rather than independently predictive. $^{3}$ For example, Kozower and colleagues ${ }^{3,4}$ have written extensively that surgical volumes are not reliable predictors of outcomes. Specifically regarding volumes of lung cancer resection and mortality, Kozower and colleagues $^{3,4}$ noted that "the apparent impact of hospital lung cancer resection volume on mortality is dependent on how volume is defined" and the statistical analysis used to evaluate hospital volume.

Despite the ongoing debate, advocacy groups, such as the Leapfrog Group, ${ }^{5}$ have proposed that hospitals reevaluate their credentialing and privileging processes to comply with minimum volume standards. This culminated in 2015 with the Take the Volume Pledge campaign. Take the Volume Pledge was a combined effort of large hospital systems to bring the notion of an association between outcomes and hospital surgical volume to national attention. It further advocated that those hospitals not meeting these volume standards should cease performing the particular procedures listed in the pledge. As has been

TABLE 5. Outcomes (lung resections)

\begin{tabular}{|c|c|c|c|c|c|c|}
\hline & \multicolumn{6}{|c|}{ Lung resections } \\
\hline & \multicolumn{4}{|c|}{ Propensity score matched } & \multicolumn{2}{|c|}{ Conditional logistic regression } \\
\hline & \multirow{2}{*}{$\begin{array}{c}\text { Low volume: } \\
\text { 1-39 surgeries/y }\end{array}$} & \multicolumn{3}{|c|}{ High volume: } & \multirow{2}{*}{$\begin{array}{c}\text { Odds ratio } \\
(95 \% \text { Confidence interval })\end{array}$} & \multirow{2}{*}{$\begin{array}{r}P \\
\text { valu }\end{array}$} \\
\hline & & $\% *$ & $40+$ surgeries $/ y$ & $\%$ & & \\
\hline Total & 6349 & 50.0 & 6349 & 50.0 & & \\
\hline In-hospital mortality & 134 & 2.1 & 113 & 1.8 & $1.19(0.93-1.54)$ & .17 \\
\hline Length of stay $\dagger$ & $6(4-9)$ & & 4-8) & & & $<.01$ \\
\hline Total charges adjusted to 2016 dollars $\dagger$ & $82,529(54,953-126,991)$ & & $997(51,254-104,8$ & & & $<.01$ \\
\hline \multicolumn{7}{|l|}{ Cardiovascular } \\
\hline Any cardiovascular complication & 1075 & 16.9 & 1087 & 17.1 & $0.99(0.90-1.08)$ & .78 \\
\hline \multicolumn{7}{|l|}{ Pulmonary } \\
\hline Any pulmonary complication & 2224 & 35.0 & 2129 & 33.5 & $1.07(0.99-1.15)$ & .07 \\
\hline Pneumonia & 364 & 5.7 & 342 & 5.4 & $1.07(0.92-1.24)$ & .40 \\
\hline Postoperative acute respiratory insufficiency & 382 & 6.0 & 440 & 6.9 & $0.86(0.75-0.99)$ & .04 \\
\hline \multicolumn{7}{|l|}{ Infectious } \\
\hline Any infectious complication & 310 & 4.9 & 266 & 4.2 & $1.18(1.00-1.40)$ & .06 \\
\hline Sepsis/shock & 149 & 2.4 & 115 & 1.8 & $1.31(1.02-1.68)$ & .03 \\
\hline \multicolumn{7}{|l|}{ Intraoperative } \\
\hline Any intraoperative complication & 180 & 2.8 & 181 & 2.9 & $0.99(0.81-1.22)$ & .96 \\
\hline Presence of any complication & 2848 & 44.9 & 2817 & 44.4 & $1.02(0.95-1.10)$ & .54 \\
\hline Total complications $\dagger$ & $0(0-1)$ & & $0-1)$ & & & .26 \\
\hline
\end{tabular}

Values are presented as n, \%, or median (interquartile range). *Percentages may not equal 100 due to rounding. $\dagger$ Outcome is linear and not analyzed by conditional logistic regression. 


\begin{tabular}{|c|c|c|c|c|c|c|}
\hline & \multicolumn{6}{|c|}{ Esophagectomy } \\
\hline & \multicolumn{4}{|c|}{ Propensity score matched } & \multicolumn{2}{|c|}{ Conditional logistic regression } \\
\hline & \multirow{2}{*}{$\begin{array}{c}\text { Low volume: } \\
1-19 \text { surgeries/y }\end{array}$} & \multicolumn{3}{|c|}{ High volume: } & \multirow{2}{*}{$\begin{array}{c}\text { Odds ratio } \\
(95 \% \text { Confidence interval })\end{array}$} & \multirow{2}{*}{$\begin{array}{c}P \\
\text { value }\end{array}$} \\
\hline & & $\% *$ & $40+$ surgeries $/ y$ & $\%$ & & \\
\hline Total & 376 & 66.7 & 188 & 33.3 & & \\
\hline In-hospital mortality & 14 & 3.7 & $<11$ & & & \\
\hline Length of stay $\dagger$ & $11.50(8-18)$ & & $12(9-19)$ & & & .1307 \\
\hline Total charges adjusted to 2016 dollars $\dagger$ & $146,849(99,732-283,377)$ & & $125,882(77,270-336,580)$ & & & .0348 \\
\hline \multicolumn{7}{|l|}{ Cardiovascular } \\
\hline Any cardiovascular complication & 118 & 31.4 & 50 & 26.6 & $1.28(0.86-1.91)$ & .23 \\
\hline \multicolumn{7}{|l|}{ Pulmonary } \\
\hline Any pulmonary complication & 198 & 52.7 & 92 & 48.9 & $1.16(0.82-1.63)$ & .41 \\
\hline Pneumonia & 57 & 15.2 & 33 & 17.6 & $0.84(0.53-1.34)$ & .47 \\
\hline \multicolumn{7}{|l|}{ Infectious } \\
\hline Any infectious complication & 63 & 16.8 & 32 & 17.0 & $0.98(0.62-1.55)$ & .94 \\
\hline \multicolumn{7}{|l|}{ Intraoperative } \\
\hline Any intraoperative complication & 24 & 6.4 & $<11$ & & & \\
\hline \multicolumn{7}{|l|}{ Other complications } \\
\hline Anastomotic leak & 47 & 12.5 & 20 & 10.6 & $1.19(0.69-2.07)$ & .53 \\
\hline Presence of any complication & 254 & 67.6 & 118 & 62.8 & $1.22(0.86-1.75)$ & .27 \\
\hline Total complications $\dagger$ & $1(0-3)$ & & $1(0-2)$ & & & .13 \\
\hline
\end{tabular}

Values are presented as n, \%, or median (interquartile range). *Percentages may not equal 100 due to rounding. $\dagger$ Outcome is linear and not analyzed by conditional logistic regression.

noted by others, ${ }^{7,15}$ there was considerable controversy related to the pledge. Of particular concern is the methodology of choosing cutpoints under which a hospital is considered low volume.

Because major thoracic surgical oncology procedures are considered in the Take the Volume Pledge, we elected to evaluate the Leapfrog Group's proposed cutpoints to determine whether they do correlate with surgical outcomes. By performing both an unmatched analysis and a propensity-matched analysis for patients operated upon in low- and high-volume hospitals, our current study is an important contribution to a growing body of thoracic surgery literature.

For our study, major lung resection included both lobectomy and pneumonectomy. Using unmatched patient data as many previous studies have done, it would appear that both in-hospital mortality and postoperative complications are higher in low-volume centers compared with high-volume centers. However, in our study, there were important demographic differences between these 2 groups that likely also influenced outcomes. Patients at low-volume centers were much more likely to be older, be minorities, live in ZIP codes in the lowest median income quartile of their state, have Medicare/Medicaid, and have chronic obstructive pulmonary disease. These represent significant differences in patient populations known to adversely influence outcomes and could certainly bias the data against low-volume hospitals. Once we accounted for such demographic factors by propensity matching, differences in outcomes attributable to a volume effect were no longer present. Following major lung resection, there was no significant difference between low- and high-volume centers for either in-hospital mortality or for postoperative complications in the propensity-matched data.

The data were similar following esophagectomy. In the unmatched data, there were no significant differences between low- and high-volume centers regarding in-hospital mortality. However, several postoperative complications were higher at low-volume centers and could potentially be attributed to a volume effect. However, although there were no significant differences in patient age or income between low- and high-volume centers, patients undergoing esophagectomy at low-volume centers were still more likely to be miniorities, have Medicare/Medicaid, and have major weight loss on admission. After propensity matching patients undergoing esophagectomy, no difference was apparent in postoperative mortality, complications, or median LOS.

Apart from purely surgical outcomes, there were important differences between the 2 groups, which may have implications for broad health care policy discussions. In the unmatched data for lung resection patients, LOS was significantly longer for the low-volume hospitals. Median hospital charges were also significantly higher in the unmatched data for lung resection patients at low-volume 
centers. Although these differences remained even in the propensity-matched analysis, clustering at the hospitallevel removed any differences in charges based on volume. Although LOS was not significantly different for patients undergoing esophagectomy at high- or low-volume centers, median hospital charges were significantly higher in the low-volume centers in both the unmatched and propensity-matched data. However, this effect disappeared when accounting for clustering at the hospital level. Total charges do not differ between high- and low-volume centers.

It is remarkable that the majority of patients in our study underwent major lung resection or esophagectomy at low-volume centers. This was particularly dramatic for esophagectomy patients, among whom $82 \%$ of patients underwent esophagectomy at a low-volume center. These findings suggest that although centers may be deemed low volume, collectively they provide a significant proportion of the cancer care for patients in the states studied. This highlights issues with access to care. Recent articles have suggested that traveling to tertiary centers to receive care may place significant burdens upon patients. ${ }^{14}$ This would appear to be especially true given the demographic characteristics we found in patients receiving care at low-volume centers. Mandating cancer care to highervolume centers could be a significant burden on these patients and could potentially adversely influence their care. It is our opinion that these competing needs will have to be balanced in future health care policy decisions.

Our study has some limitations. Because our study was conducted over a 3-year period, it is possible for a hospital to be included in different volume categories during the study period. Although this is a limitation of the current study, it highlights the challenges associated with strict volume cutpoints. Additionally, administrative databases are not optimized for evaluation and interpretation of clinical results or for quality improvement. Unfortunately, the SID database does not contain any information pertaining to management algorithms following lung resection or esophagectomy. Important surgeon and hospital characteristics may have been missed, including surgical volume by provider. Similarly, ICD-9-CM codes lack detailed standard clinical definitions that can be universally applied and are thus open to coding interpretation. These are certainly limitations of the study, along with the lack of cancer staging information. Outcomes following surgery for cancer should not focus solely on immediate complications and mortality, but should also address long-term quality of life and cancer survival. Given the constraints of the data we were unable to comment upon this in our current study. However, these administrative data regarding perioperative outcomes are widely generalizable and not restricted to the outcomes of specialized, experienced centers. Additionally, we believe that by propensity matching patient cohorts we were able to overcome methodologic limitations of several previous studies demonstrating a volume effect with lung resection and esophagectomy.

\section{CONCLUSIONS}

Volume standards may be an important component of surgical outcomes, but other factors- such as race, income, comorbidities, and access to care-are also involved. After propensity matching, many volume effects were negated. Our study illustrates the complexity of health care delivery and that low volumes of particular procedures is unlikely an isolated factor leading to inferior outcomes. A majority of our patients received care at low-volume centers. Restricting access to such centers could potentially significantly influence health care delivery. Given the complexity of the situation and its potential to change the practice of thoracic surgery and the care delivered to patients, we believe that major changes in health care policy in this arena should be led by national surgical societies.

\section{Conflict of Interest Statement}

Authors have nothing to disclose with regard to commercial support.

\section{References}

1. Bach PB, Cramer LD, Schrag D, Downey RJ, Gelfand SE, Begg CB. The influence of hospital volume on survival after resection for lung cancer. $N$ Engl J Med. 2001:345:181-8

2. Birkmeyer JD, Siewers AE, Finlayson EV, Stukel TA, Lucas FL, Batista I, et al. Hospital volume and surgical mortality in the United States. N Engl J Med. 2002 346:1128-37.

3. Kozower BD, Stukenborg GJ. The relationship between hospital lung cancer resection volume and patient mortality risk. Ann Surg. 2011;254 1032-7.

4. Meguid RA, Weiss ES, Chang DS, Brock MV, Yang SC. The effect of volume on esophageal cancer resections: what constitutes acceptable resection volumes for centers of excellence? J Thorac Cardiovasc Surg. 2009;137:23-9.

5. LaPar DJ, Kron IL, Jones DR, Stukenborg GJ, Kozower BD. Hospital procedure volume should not be used as a measure of surgical quality. Ann Surg. 2012;256: 606-15.

6. Milstein A, Galvin RS, Delbanco SF, Salber P, Buck CR Jr. Improving the safety of health care: the leapfrog initiative. Eff Clin Pract. 2000;3:313-6.

7. Urbach DR. Pledging to eliminate low-volume surgery. N Engl J Med. 2015;373: 1388-90.

8. Proposed changes to the 2017 Leapfrog Hospital Survey. Available at: http://www leapfroggroup.org/sites/default/files/Files/Proposed-Changes-2017-Leapfrog-Hos pital-Survey-Final.pdf. Accessed March 20, 2017.

9. 2013 Healthcare Cost and Utilization Project. Available at: http://www.hcup-us ahrq.gov/sidoverview.jsp. Accessed March 20, 2017.

10. Romano PS, Chan BK, Schembri ME, Rainwater JA. Can administrative data be used to compare postoperative complication rates across hospitals? Med Care. 2002;40:856-67.

11. Lawson EH, Louie R, Zingmond DS, Brook RH, Hall BL, Han L, et al. A comparison of clinical registry versus administrative claims data for reporting of 30day surgical complications. Ann Surg. 2012;256:973-81.

12. Quan H, Li B, Couris CM, Fushimi K, Graham P, Hider P, et al. Updating and validating the Charlson comorbidity index and score for risk adjustment in hospital discharge abstracts using data from 6 countries. Am J Epidemiol. 2011;173: 676-82.

13. Khuri SF, Daley J, Henderson W, Hur K, Hossain M, Soybel D, et al. Relation of surgical volume to outcome in eight common operations: results from 
the VA National Surgical Quality Improvement Program. Ann Surg. 1999; 230:414-29.

14. Begg CB, Cramer LD, Hoskins WJ, Brennan MF. Impact of hospital volume on operative mortality for major cancer surgery. JAMA. 1998;280:1747-51.

15. Blanco BA, Kothari AN, Blackwell RH, Brownlee SA, Yau RM, Attisha JP, et al. "Take the Volume Pledge" may result in disparity in access to care. Surgery. 2017; 161:837-45.

16. Finks JF, Osborne NH, Birkmeyer JD. trends in hospital volume and operative mortality for high-risk surgery. N Engl J Med. 2011;364:2128-37.

17. Learn PRA, Bach PB. A decade of mortality reductions in major oncologic surgery: the impact of centralization and quality improvement. Med Care. 2010;48:1041-9.
18. Sudhir S, McLeod R, Irish J, Burns J, Hunter A, Meertens E, et al. Early results after regionalization of thoracic surgical practice in a single-payer system. Ann Thorac Surg. 2013;95:472-9.

19. Ogola GE, Haider A, Shafi S. Hospitals with higher volumes of emergency general surgery patients achieve lower mortality rates: a case for establishing designated centers for emergency general surgery. J Trauma Acute Care Surg. 2017;82:497-504.

Key Words: minimal volume standards, outcomes, lung surgery, esophageal surgery 
TABLE E1. Intraoperative/procedural complications

\section{ICD-9-CM codes}

\begin{tabular}{|c|c|}
\hline \multicolumn{2}{|l|}{ Cardiovascular } \\
\hline Supraventicular arrhythmia & $(427.31,427.3,427.32,427.0)$ \\
\hline Myocardial infarction & $(410-10.9,411.81,413-413.9)$ \\
\hline Postoperative stroke & $(997.02,430-435, \mathrm{~V} 12.54, \mathrm{~V} 17.1)$ \\
\hline Deep venous thrombosis & $(451.1,451.2,451.81,451.9,453.2,453.8,453.9,453.40-453.42)$ \\
\hline Pulmonary embolism & $(415.1,415.11,415.12,415.39)$ \\
\hline \multicolumn{2}{|l|}{ Pulmonary } \\
\hline Pneumonia & $(486,481,482.8,482.3,482.9,481,482.0-482.2,482.41,482.49,482.83,997.31)$ \\
\hline Postoperative acute respiratory insufficiency & $(518.5)$ \\
\hline Postoperative acute pneumothorax & $(512.1)$ \\
\hline Postoperative pulmonary edema & $(518.4)$ \\
\hline Pulmonary collapse & $(518.0)$ \\
\hline Empyema with and without fistula & $(510.0,510.9)$ \\
\hline Mechanical ventilation & $(96.70,96.71,96.72)$ \\
\hline Noninvasive ventilation & (93.90) \\
\hline Tracheostomy & $(31.1-31.29,31.2)$ \\
\hline Sarcoidosis & $(135)$ \\
\hline \multicolumn{2}{|l|}{ Infectious } \\
\hline Sepsis/shock & $(995.91,038,995.92,999.3,998.0)$ \\
\hline Urinary tract infection & $(599.0,590.9)$ \\
\hline Postoperative wound infection & $(998.51,998.59)$ \\
\hline Fever & $(780.60)$ \\
\hline \multicolumn{2}{|l|}{ Intraoperative complication } \\
\hline Accidental puncture or laceration complicating surgery & $(998.2)$ \\
\hline Bleeding complicating procedure & (998.11) \\
\hline \multicolumn{2}{|l|}{ Other } \\
\hline Anastomotic leak (for esophagectomy) & $(997.4)$ \\
\hline
\end{tabular}

